\title{
BAYESIAN PARAMETER IDENTIFICATION OF LATTICE DISCRETE PARTICLE MODEL FOR CONCRETE
}

\author{
E. Janouchová ${ }^{*}$ A. Kučerová ${ }^{* *}$, J. Sýkora ${ }^{* * *}$, J. Vorel $^{\dagger}$, R. Wendner ${ }^{\ddagger}$
}

\begin{abstract}
The investigated lattice discrete particle model allows to simulate the failure behaviour of concrete. The main characteristic of this model is its response dependence on randomly generated geometrical features of material internal structure according to the basic concrete properties and the size distribution of the aggregates. This makes the model stochastic and the corresponding calibration difficult. On top of this, the model simulation is computationally demanding which limits usage of the full numerical model in an identification procedure. To overcome these obstacles, the polynomial chaos based surrogate model is employed. This approximation eradicates the original model stochasticity, but the corresponding uncertainty can be estimated from the approximation error. The quantified uncertainty is then involved in the Bayesian parameter inference based on Markov chain Monte Carlo sampling with use of cheap surrogate evaluations. The proposed identification procedure is verified on a basis of synthetic experimental data set from notched three-point-bending tests and cube compression tests.
\end{abstract}

Keywords: lattice discrete particle model, stochastic modelling, polynomial chaos expansion, Bayesian inference, Markov chain Monte Carlo

\section{Introduction}

Lattice or particle formulations of models are suitable for description of macroscopic behaviour of quasibrittle materials. The medium is discretized "a priori" according to an idealization of its internal structure. The particles or lattice are defined by geometrical parameters which equip these type of formulations with inherent characteristic lengths and they have the intrinsic ability of simulating the geometrical features of material internal structure. This enables to accurately simulate a damage initiation and crack propagation at various length scales, but the computational demands are significant. Here we employ the so-called Lattice Discrete Particle Model (LDPM) recently proposed by Cusatis et al. (2011a). The LDPM was calibrated and validated against both quasi-static and dynamic loading conditions and it was demonstrated to possess superior predictive capability, see Cusatis et al. (2011b). However, the calibration procedure was performed on a basis of hand-fitting, which makes any further practical applications of the model difficult.

Here we present the Bayesian identification of the model parameters from synthetic experimental data corresponding to notched three-point-bending tests and cube compression tests. The Bayesian approach solves the inverse problem as well-possed and quantifies posterior uncertainty in parameters by combining a prior knowledge about the realistic parameter values and uncertainty contained in measurement errors. In more details, we obtain the posterior distributions by robust Markov chain Monte Carlo sampling, where

* Ing. Eliška Janouchová: Faculty of Civil Engineering, CTU in Prague; Thákurova 7/2077; 166 29, Prague; CZ, eliska.janouchova@fsv.cvut.cz

** Ing. Anna Kučerová, Ph.D.: Faculty of Civil Engineering, CTU in Prague; Thákurova 7/2077; 166 29, Prague; CZ, Anna.Kucerova@cvut.cz

*** Ing. Jan Sýkora, Ph.D.: Faculty of Civil Engineering, CTU in Prague; Thákurova 7/2077; 166 29, Prague; CZ, jan.sykora.1@fsv.cvut.cz

$\dagger \quad$ doc. Ing. Jan Vorel, Ph.D.: Faculty of Civil Engineering, CTU in Prague; Thákurova 7/2077; 166 29, Prague; CZ, jan.vorel@fsv.cvut.cz

‡ Priv.-Doz. Dipl.-Ing. Dr. Roman Wendner: Christian Doppler Laboratory LiCRoFast, Institute of Structural Engineering, University of Natural Resources and Life Sciences; Peter-Jordan-Straße 82; 1190, Vienna; Austria, roman.wendner@boku.ac.at 
the computational burden, arising from repeated model simulations, is overcome by using a polynomial chaos-based surrogate of the LDPM. Moreover, this model approximation allows to eradicate the original LDPM stochasticity and involve the corresponding uncertainty estimated from the approximation error to the identification procedure.

\section{Lattice discrete particle model}

In this paper the LDPM is employed to accurately describe the macroscopic behaviour of concrete in elastic, fracturing, softening, and hardening regimes (Cusatis et al. $(2011 \mathrm{a}, \mathrm{b})$ ). The examined material model is based on lattice or particle formulations in which materials are discretized "a priori" according to an idealization of their internal structure. In the present study the basic material properties of the tested concrete mix are kept constant for all simulations. Note that these parameters influence the generation of concrete meso-structure, see Table 1. However, the seed number, governing the sampling of cumulative distribution

Tab. 1: Values of parameters governing the generation of concrete meso-structure

\begin{tabular}{llll}
\hline Material property & & Unit & Value \\
\hline Minimum particle size & $d_{0}$ & $\mathrm{~mm}$ & 4 \\
Maximum particle size & $d_{a}$ & $\mathrm{~mm}$ & 16 \\
Cement content & $c$ & $\mathrm{~kg} / \mathrm{m}^{3}$ & 240 \\
Water to cement ratio & $w / c$ & - & 0.83 \\
Aggregate to cement & $a / c$ & - & 8.83 \\
Fuller coefficient & $n_{F}$ & - & 0.5 \\
Concrete density & $\rho$ & $\mathrm{kg} / \mathrm{m}^{3}$ & 2400 \\
\hline
\end{tabular}

function of concrete granulometric distributions by a random number generator, is kept random. The model response is influenced by the specific distribution of particles which causes stochasticity of the model.

The parameters of the mathematical model which are kept constant or calibrated by the identification procedure of prescribed uniform prior distribution are summarised in Table 2.

\begin{tabular}{llll} 
Tab. 2: Values of material model parameters used in the numerical simulations \\
\cline { 2 - 4 } Material property & & Unit & Value (range) \\
\hline Normal modulus & $E_{0}$ & $\mathrm{MPa}$ & $20000-70000$ \\
Shear-normal coupling & $\alpha$ & - & $0.2-0.3$ \\
Tensile strength & $\sigma_{t}$ & $\mathrm{MPa}$ & $1.5-5$ \\
Tensile characteristic length & $l_{t}$ & $\mathrm{~mm}$ & $50-300$ \\
Softening exponent & $n_{t}$ & - & $0.1-1$ \\
Shear/strength ratio & $\sigma_{s} / \sigma_{t}$ & - & $1.5-8$ \\
Initial friction & $\mu_{0}$ & - & $0.001-0.5$ \\
Compressive strength & $\sigma_{c 0}$ & $\mathrm{MPa}$ & $\sigma_{c 0}=40 \sigma_{t}$ \\
Transitional stress & $\sigma_{N 0}$ & $\mathrm{MPa}$ & $\sigma_{N 0}=240 \sigma_{t}$ \\
\hline Initial hardening modulus ratio & $H_{c 0} / E 0$ & - & 0.4 \\
Transitional strain ratio & $\kappa_{c 0}$ & - & 4 \\
Deviatoric strain threshold ratio & $\kappa_{c 1}$ & - & 1.0 \\
Deviatoric damage parameter & $\kappa_{c 2}$ & - & 5.0 \\
Asymptotic friction & $\mu_{\infty}$ & - & 0.0 \\
Densification ratio & $E_{d} / E_{0}$ & - & 1.0 \\
Volumetric-deviatoric coupling & $\beta$ & - & 0 \\
\hline
\end{tabular}

\section{Identification procedure}

The identification of seven material model parameters for concrete is based on two types of experiments, specifically a uniaxial compression test and a notched three-point-bending test. In order to verify the pro- 
posed identification method, the synthetic experimental data are used in a form of 10 data sets with three repetitions of each test. The corresponding design of experiments is obtained by a stratified procedure called Latin hypercube sampling (LHS) which is able to respect the prescribed probability distributions (Janouchová and Kučerová (2013)). For convenience and readability, data are presented in terms of nominal stress $\sigma_{N}$ and nominal strain $\varepsilon_{N}$. The most traditional tests to characterise concrete is the compression test performed on cubes of $150 \mathrm{~mm}$ side length. The nominal values are defined as

$$
\sigma_{N}=\frac{F}{a^{2}} \text { and } \varepsilon_{N}=\frac{u}{a}
$$

where $F$ is the applied load, $u$ denotes the load point displacement $a$ is a side length. The fracture properties of concrete are characterised by means of the notched three-point-bending test. The nominal stress and strain are

$$
\sigma_{N}=\frac{3 F l}{d h^{2}} \text { and } \varepsilon_{N}=\frac{C M O D}{h}
$$

where $l$ stands for the beam span, $h, d$ are the beam height and width, respectively. $C M O D$ is the crack mouth opening displacement measured over the notch.

Moreover, to eliminate the error in measurement caused by the testing machine stiffness, the inelastic part of the strain is used for the model calibration

$$
\varepsilon_{N}^{\text {inel }}=\varepsilon_{N}-\sigma_{N}(1 / K)
$$

where $K$ is the corresponding elastic stiffness. To capture the elastic properties of the model, the initial elastic part of the cube compression test is utilised.

In order to make the identification process feasible, we employ polynomial chaos expansion (PCE) for the approximation of the model response in the stochastic space (Marzouk et al. (2007)). PCE can be used to approximate the response with respect to the probability distribution of the random variables. The convergence of the approximation error with the increasing number of polynomial terms is optimal in case of orthogonal polynomials of a special type corresponding to the probability distribution of the underlying variables (Xiu and Karniadakis (2002)). In particular, we employ Legendre polynomials of the second order associated with the uniform distribution. The PCE coefficients are computed with help of linear regression (Blatman and Sudret (2010a)), which is based on a set of 400 model simulations drawn by LHS.

Probabilistic parameter estimation allowing to update the initial knowledge about the parameter values by processing the experimental data is based on Bayes' formula (Tarantola (2005)). The updated joint probability distribution of the parameters is formulated as a product of prescribed uniform prior distribution with bounds given in Table 2 and likelihood function arising from the uncertainties which correspond to the experimental errors and theoretical errors caused by approximating the stochastic LDPM by deterministic PCE-based surrogate. The experimental errors are based on expert knowledge and considered as independent normally distributed random variables with zeros mean values and prescribed standard deviations. Specifically, in the identification process we consider from the compression test the measured stress $\sigma_{N}$ discretized into 200 strain steps with the error $\varepsilon \sim N\left(0,1.3^{2}\right)$ and elastic stiffness $K$ with error $\varepsilon \sim N\left(0,150^{2}\right)$, from the notched three-point-bending test the measured stress $\sigma_{N}$ discretized into 200 strain steps with the error $\varepsilon \sim N\left(0,0.3^{2}\right)$. On the other side, the theoretical errors are dependent normally distributed random variables whose covariance matrix is estimated on a basis of approximation errors for training set of model simulations. To obtain the posterior distribution, we employ Markov chain Monte Carlo sampling method (Gilks et al. (2005)). The Metropolis algorithm (Spall (2003)) is used to construct the chain of a million posterior samples for each data set.

Fig. 1 shows the obtained results of executed identification processes in the sense of model responses. There are 400 training model responses depicted by grey colour in the background. For the sake of clarity, the only five out of ten data sets are depicted in the graphs. The data corresponding to one data set are displayed by one colour in both cases of experiment types. The solid lines shows the synthetic experimental data and the filled areas correspond to the identification results. More specifically, the displayed areas are defined by spread equal to identified mean values \pm standard deviations, so they contains stochasticity of the LDPM as well as uncertainties rising from experimental measurements and predict the regions of highly probable occurrence of reality. It is obvious that the presented identification procedure provides results, in a form of joint probability distribution of model parameters, with very good predictive ability for the LDPM. On 
the basis of these results, the proposed identification method is verified to be efficiently used for the LDPM calibration.
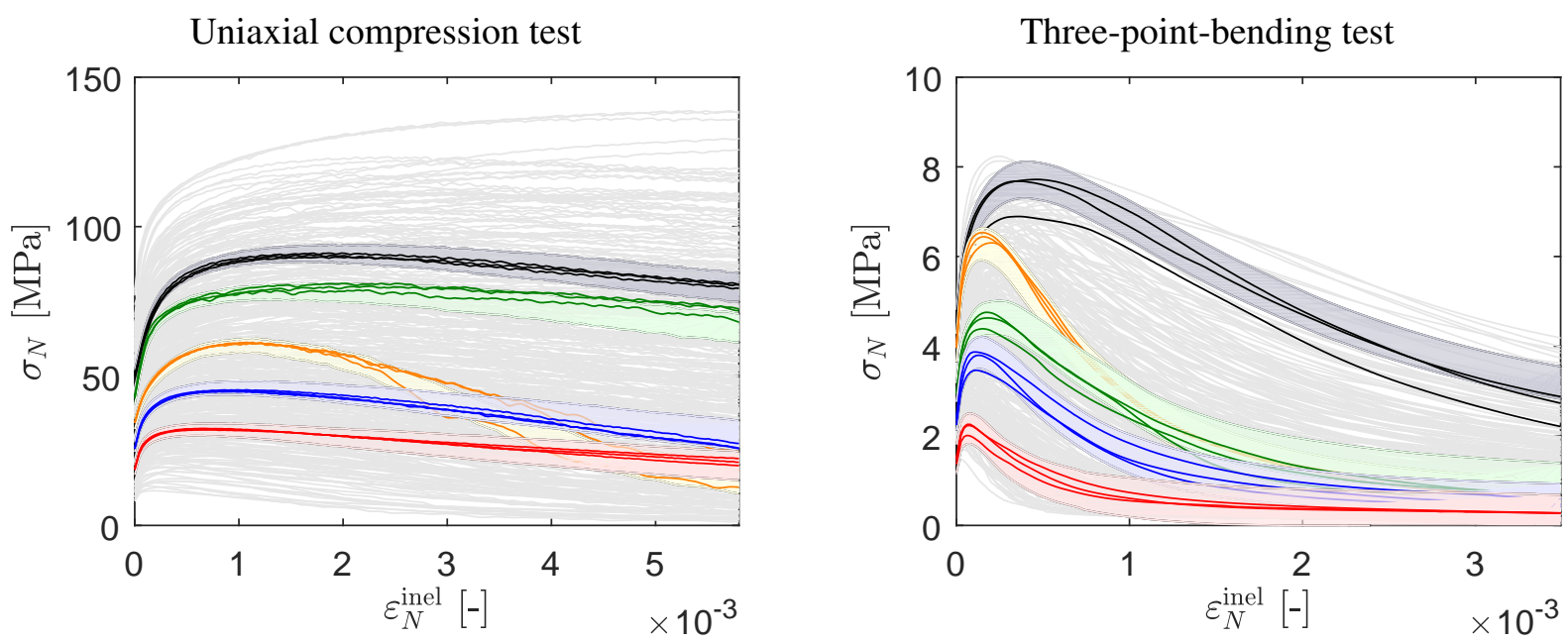

Fig. 1: Gray model output curves for construction of approximation vs. colourful results of verification. Solid lines are synthetic experimental data while filled areas represent identified regions of high prediction probability. Each colour corresponds to one verification data set.

\section{Conclusions}

This contribution deals with probabilistic parameter identification of lattice discrete particle model for concrete. The presented identification procedure is based on polynomial approximation of the stochastic model response and Bayesian inference of the underlying model parameters. The proposed method allows to calibrate the LDPM from a uniaxial compression test and a notched three-point-bending test, which was successfully verified on the basis of ten sets of synthetic experimental data. The future work includes method validation based on real experimental data.

\section{Acknowledgments}

The financial support of the Czech Science Foundation (Project No. 16-11473Y) is gratefully acknowledged.

\section{References}

Blatman, G., Sudret. B. An adaptive algorithm to build up sparse polynomial chaos expansions for stochastic finite element analysis. Probabilistic Engineering Mechanics, 25(2):183-197, 2010a.

Cusatis, G., Pelessone, D., Mencarelli, A. Lattice Discrete Particle Model (LDPM) for failure behavior of concrete. I: Theory. Cement and Concrete Composites, 33(9):881-890, 2011.

Cusatis, G., Mencarelli, A., Pelessone, D., Baylot, J. Lattice Discrete Particle Model (LDPM) for failure behavior of concrete. II: Calibration and validation. Cement and Concrete Composites, 33(9):891-905, 2011.

Gilks, W. R., et al. Markov Chain Monte Carlo. In Encyclopedia of Biostatistics, Chichester, UK: John Wiley, 75(3):333-357, 2005.

Janouchová, E., Kučerová, A. Competitive Comparison of Optimal Designs of Experiments for Sampling-based Sensitivity Analysis. Computers \& Structures, 124:47-60, 2013.

Marzouk, Y. M., Najm, H. N., Rahn, L. A. Stochastic spectral methods for efficient Bayesian solution of inverse problems. Journal of Computational Physics 224:560-586, 2007.

Spall, J. C. Estimation via Markov Chain Monte Carlo. IEEE Control Systems Magazine, 23(2):34-45, 2003.

Tarantola, A. Inverse problem theory and methods for model parameter estimation. Philadelphia, PA: Society for Industrial and Applied Mathematics, 348 p., 2005.

Xiu, D., Karniadakis, G. E. The Wiener-Askey Polynomial Chaos for Stochastic Differential Equations. SIAM Journal on Scientific Computing, 24(2):619-644, 2002. 